\title{
SELECTIVE BIOHERBICIDAL POTENTIAL OF DELONIX REGIA ALLELOPATHIC LEAF EXTRACT ON GERMINATION AND SEEDLING GROWTH OF FIELD BINDWEED AND WHEAT
}

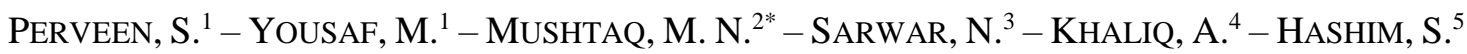 \\ ${ }^{1}$ Department of Chemistry, Government College University, Faisalabad, Pakistan \\ ${ }^{2}$ University of Agriculture Faisalabad, Sub Campus Burewala, Pakistan \\ ${ }^{3}$ Plant Protection Division, Nuclear Institute for Agriculture and Biology, Faisalabad, Pakistan \\ ${ }^{4}$ Department of Agronomy, University of Agriculture, Faisalabad, Pakistan \\ ${ }^{5}$ Department of Weed Science, University of Agriculture, Peshawar, Pakistan \\ *Corresponding author \\ e-mail:mnmushtaq@gmail.com \\ (Received $2^{\text {nd }}$ Sep 2018; accepted $1^{\text {st }}$ Nov 2018)
}

\begin{abstract}
Due to increased risk attached with indiscriminate use of synthetic herbicides, researchers are looking for alternative weed control strategies. Allelopathy is a natural phenomenon that might be exploited for biological weed management in field crops. Allelopathic plant water extracts may provide selective growth inhibition of the weeds. The present studies were conducted to investigate the possible selective phytotoxic effect of Delonix regia aqueous, ethyl acetate and lyophilized leaf extract against field bindweed (Convolvulus arvensis L.) and wheat (Triticum aestivum L.). The results revealed that lower to medium aqueous (2.5 and 5\%) and ethyl acetate (50,500 and $1000 \mathrm{ppm}$ ) leaf extract concentrations inhibited filed bindweed germination, root length, shoot length and seedling dry biomass by $35-48 \%, 42-59 \%, 45-46 \%$ and $52-56 \%$ respectively, while no significant phytotoxic effect was observed on wheat at these concentrations. It is concluded that lower to medium concentrations of Delonix regia leaf extract might be safely utilized for natural control of field bindweed in wheat, reducing reliance upon synthetic herbicides.
\end{abstract}

Keywords: allelopathy, biocontrol, Convolvulus arvensis, phytotoxic, Triticum aestivum

\section{Introduction}

Use of synthetic herbicides is an efficient way to control weeds in horticultural and agricultural field crops and herbicide use has been increased many folds in the last few decades. The worldwide consumption of herbicides is about 0.95 million tons per year, which accounts for $47.5 \%$ of the total pesticides used (De et al., 2014). Indiscriminate use of herbicides is not only creating herbicide resistant weeds but also posing serious threat to the environment (Polyrakis, 2009). At present, there are 255 herbicide resistant weed species (148 dicots and 107 monocots) reported in 92 crops and 70 countries (Heap, 2018). Moreover, herbicides also contaminate air, soil, surface water, ground water, non-target organisms and herbicide residues in the food has increased risk of human diseases especially in the workers at farm level and/or in agricultural industries (Aktar et al., 2009; Polyrakis, 2009). Therefore, there is a growing public and scientific concern regarding herbicide use and researchers are looking for alternative ways for weed management worldwide. 
Higher plants produce secondary metabolites, which are known as allelochemicals, through a phenomenon called allelopathy. The allelochemicals may inhibit growth of the other plants including weeds (Albuquerque et al., 2011). Many plant species have been well documented as allelopathic, producing allelochemicals (Cheng and Cheng, 2015). There are different strategies for using allelopathic plants for weed management. Among the strategies, use of allelopathic plant extracts, as an alternative to synthetic herbicides, is a viable option for managing weeds (Iqbal et al., 2009; Khaliq et al., 2012; Mushtaq et al., 2010).

For effective weed management, allelopathic plant water extracts are required to be selectively phytotoxic against weeds and safe on crops (Haq et al., 2010). However, they might also inhibit germination and growth of crops (Mushtaq et al., 2013). Therefore, it is pertinent to evaluate selective phytotoxic effect of allelopathic plant water extracts against weeds in comparison with crops.

Delonix regia (Boj.) Raf. (flamboyant) is a 10-15 m tall tree native to Madagascar and spread world over in tropical and subtropical regions (Fig. 1). It belongs to biological nitrogen fixing family (Fabaceae) of plants. It is a decorative tree with beautiful flowers and used as shade, shelter, timber, fuel and in apiculture (Orwa et al., 2009). Previously, it has been reported that Delonix regia substantially inhibited weeds under its canopy. Aqueous extract of Delonix regia inhibited growth of Centella asiatica and Isachne nipponensis up to 70\%. Phytotoxins present in Delonix regia were identified as chlorogenic acid, 4-hydroxybenzoic, 3,4-dihydroxybenzoic, 3,5dinitrobenzoic, L-azetidine-2-carboxylic 3,4-dihydroxybenzaldehyde, 3,4dihydroxycinnamic and gallic acid (Chou and Leu, 1992).

Field bindweed (Convolvulus arvensis L.) is a dicot perennial broadleaf weed which is considered one of the world's top 10 worst weeds by agriculturists and horticulturists (Holm et al., 1977; Skinner et al., 2000). It is an invasive species and problematic weed of many field crops including wheat. Uncontrolled densities of field bindweed may reduce yield of wheat crop by 56\%, and it is very difficult to control even with the synthetic herbicides (Black et al., 1994). The present study was conducted to compare phytotoxic protentional and selectivity of Delonix regia aqueous, ethyl acetate and lyophilized extracts on germination and early seedling growth of field bindweed and wheat.

\section{Materials and methods}

\section{Preparation of extracts}

Delonix regia leaves were collected from trees at University of Agriculture, Faisalabad Pakistan. The extraction procedure was modified from previously described (Perveen et al., 2014) unless stated otherwise. The leaves were dried under shade and were ground to fine powder. The aqueous extract was prepared by soaking $10 \mathrm{~g}$ leaf powder in $100 \mathrm{ml}$ distilled water. The suspension was placed in an ultrasonic device for 30 min (Sheng et al., 2012). The extract was filtered through two layers of cheesecloth to obtain extract concentration of $10 \%(10 \mathrm{~g} / 100 \mathrm{ml})$ and was diluted with distilled water to prepare further aqueous extract dilutions $(2.5 \%, 5 \%$ and $7.5 \%)$.

For ethyl acetate and lyophilized fractions, $200 \mathrm{ml}$ of acetone was added to $200 \mathrm{ml}$ of $10 \%$ Delonix regia aqueous leaf extract. After placing at $4{ }^{\circ} \mathrm{C}$ for $24 \mathrm{~h}$ and filtration with Whatman \#1 filter paper, the acetone was allowed to evaporate with a rotary evaporator (BUCHI, Switzerland) under reduced pressure. Aliquots of ethyl acetate 
$(600 \mathrm{ml})$ were used to partition the extract 3 times sequentially. Thereafter, ethyl acetate layer was separated, and ethyl acetate was evaporated to obtain the residue. The aqueous phase was lyophilized (freeze-dried) in a Freeze Dryer (Alpha I-5, Cherist). Thus, ethyl acetate and lyophilized residue fractions were obtained as 400 and $105 \mathrm{mg}$ respectively. The stock solution of $4000 \mathrm{ppm}$ was prepared by dissolving $100 \mathrm{mg}$ of each residue in $25 \mathrm{ml}$ methanol. The stock solution (4000 ppm) was diluted to prepare 50, 500, 1000 and 2000 ppm extract.

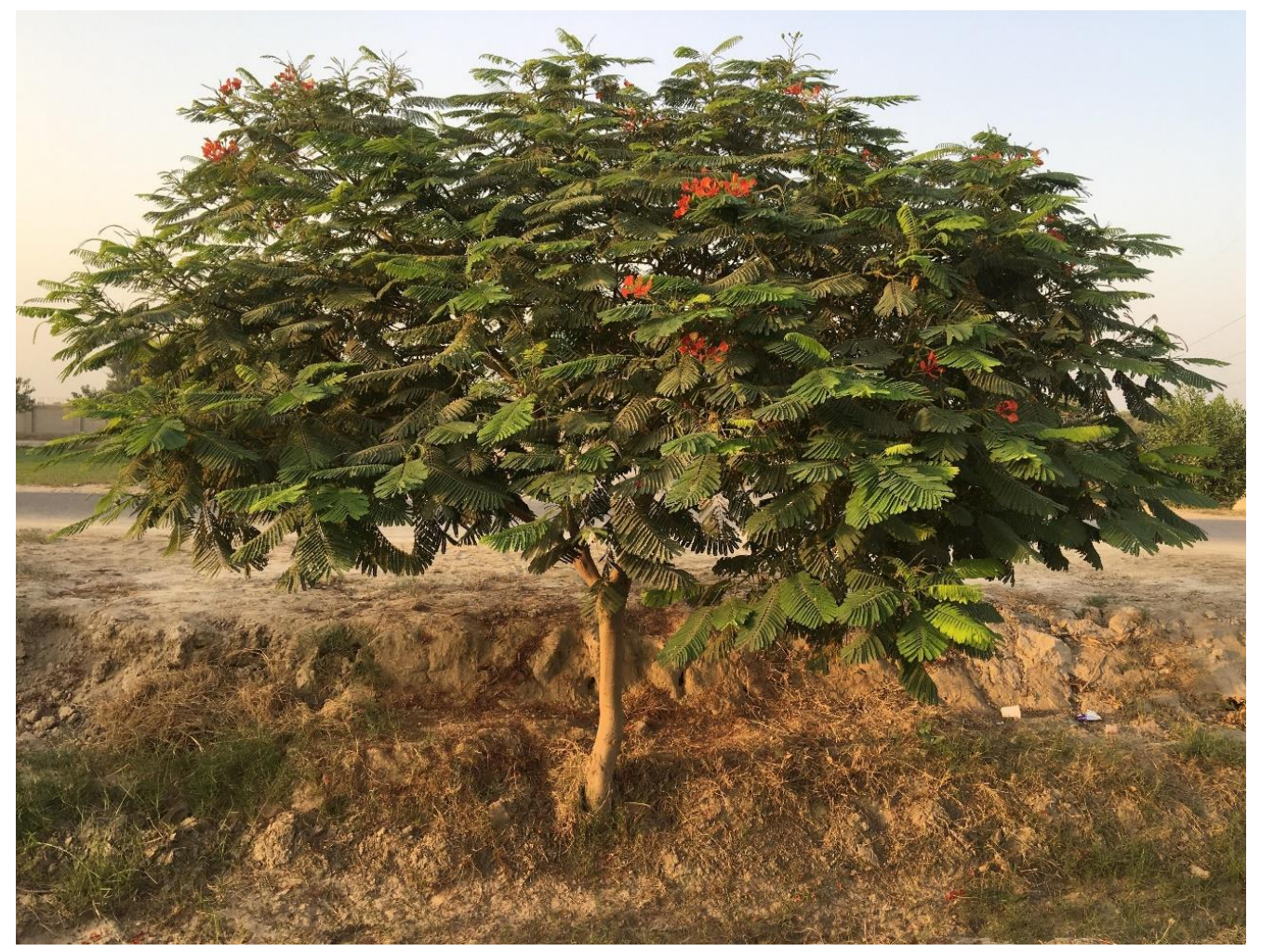

Figure 1. A young plant of Delonix regia (flamboyant) at University of Agriculture Faisalabad, Sub Campus Burewala, Pakistan. (Photo by Muhmmad Naeem Mushtaq)

\section{Bioassays}

Seeds of field bindweed and wheat were surface sterilized with $2 \%$ sodium hypochlorite for $2 \mathrm{~min}$ and were rinsed with distilled water. Each aqueous extract concentration was added $(4 \mathrm{ml})$ to sterilized petri dishes $(9.5 \mathrm{~cm})$ on Whatman \#1 filter paper. While, for ethyl acetate and lyophilized fractions, each methanolic extract $(2 \mathrm{ml})$ was added to each petri dish $(4.5 \mathrm{~cm})$ except control which received $2 \mathrm{ml}$ of pure methanol. Later, methanol was evaporated and distilled water $(2 \mathrm{ml})$ was added to each petri dish.

Ten seeds were placed on filter paper in each petri dish and the petri dishes were covered and put in a germinator at $24 \pm 2{ }^{\circ} \mathrm{C}$ with $10 / 14 \mathrm{~h}$, light/dark. Germination of seeds were noted daily. Root and shoot lengths of field bindweed and wheat were measured 7 days after sowing. The seedlings were dried in an oven at $40^{\circ} \mathrm{C}$ till constant weight to measure dry weight. Germination index (GI), mean germination time and time to start germination were calculated as reviewed and described earlier (Ranal and Santana, 2006). The GI was calculated as following: 


$$
\mathrm{GI}=\frac{\text { Number of germinated seeds }}{\text { Days of first count }}+----+\frac{\text { Number of germinated seeds }}{\text { Days of final count }}
$$

\section{Statistical analysis}

The data were analyzed by analysis of variance and treatment means were statistically compared using Duncan's Multiple Range Test. The experiments were performed as completely randomized design with 5 replications.

\section{Results}

\section{Effect of Delonix regia aqueous leaf extract on germination of field bindweed and wheat}

Leaf extract of Delonix regia had more inhibitory effect on final germination and germination index and increased mean germination time and time to start germination in field bindweed as compared with wheat (Table 1). At 5\% concentration, field bindweed took significantly double time (3.6 days) to start germination, while there was no statistically significant effect on time to start germination for wheat crop as compared with control (Table 1). Germination index and final germination was decreased by $76 \%$ and $35 \%$ respectively as compared with control in field bindweed at $5 \%$ concentration of Delonix regia leaf extract. In case of wheat, this medium concentration (5\%) did not significantly affect germination index and final germination. At maximum concentration (10\%) of Delonix regia leaf extract, final germination of field bindweed and wheat was inhibited by $49 \%$ and $9 \%$ respectively as compared with control (Table 1).

Table 1. Effect of Delonix regia aqueous leaf extract on germination of field bindweed and wheat

\begin{tabular}{|c|c|c|c|c|c|c|c|c|}
\hline \multirow{2}{*}{$\begin{array}{c}\text { Leaf extract } \\
\text { concentration } \\
(\%)\end{array}$} & \multicolumn{2}{|c|}{$\begin{array}{c}\text { Time to start germination } \\
\text { (days) }\end{array}$} & \multicolumn{2}{|c|}{$\begin{array}{c}\text { Mean germination time } \\
\text { (days) }\end{array}$} & \multicolumn{2}{|c|}{ Germination index } & \multicolumn{2}{|c|}{ Final germination $(\%)$} \\
\hline & $\begin{array}{l}\text { Field bind } \\
\text { weed }\end{array}$ & Wheat & & Wh & & Whe & & What \\
\hline 0 & & & & & & & $57.30 \pm 1.63 \mathrm{a}$ & \\
\hline 2.5 & $\begin{array}{r}1.80 \pm \\
\quad(0\end{array}$ & $\begin{array}{r}1.0 \pm \\
(C\end{array}$ & $\begin{array}{r}3.05 \pm \\
(-\end{array}$ & $1.80 \pm$ & 7.1士 & $5 \mathrm{a}$ & $\begin{array}{r}50.60= \\
(-1\end{array}$ & (2) \\
\hline 5.0 & $\begin{array}{r}3.60 \pm \\
\quad(10\end{array}$ & $\begin{array}{r}1.0 \pm \\
(0\end{array}$ & $\begin{array}{r}3.72 \pm \\
(1\end{array}$ & $\begin{array}{r}1.78 \pm \\
(-\end{array}$ & $\begin{array}{r}1.75 \\
(-\end{array}$ & $.35 \mathrm{a}$ & $\begin{array}{r}37.30 \pm \\
(-3\end{array}$ & $\begin{array}{c}82 \pm 1.99 \mathrm{a} \\
(-5)\end{array}$ \\
\hline 7.5 & $\begin{array}{l}4.80 \pm 0.20 \mathrm{a} \\
\quad(167)\end{array}$ & $\begin{array}{c}1.0 \pm 0.0 \mathrm{~b} \\
(0)\end{array}$ & $\begin{array}{l}4.80 \pm 0.2 \mathrm{a} \\
\quad(48)\end{array}$ & $\begin{array}{l}2.21 \pm 0.19 b \\
\quad(14)\end{array}$ & $\begin{array}{c}0.96 \pm 0.04 \mathrm{c} \\
(-86)\end{array}$ & $\begin{array}{c}10.5 \pm 0.58 \mathrm{a} \\
(-5)\end{array}$ & $\begin{array}{c}30.60 \pm 1.63 \mathrm{~d} \\
(-47)\end{array}$ & $\begin{array}{l}84 \pm 2.45 \mathrm{a} \\
\quad(-2)\end{array}$ \\
\hline 10 & $\begin{array}{l}4.80 \pm 0.20 \mathrm{a} \\
(167)\end{array}$ & $\begin{array}{c}1.8 \pm 0.20 \mathrm{a} \\
(80)\end{array}$ & $\begin{array}{l}4.92 \pm 0.08 \mathrm{a} \\
\quad(51)\end{array}$ & $\begin{array}{c}3.13 \pm 0.07 \mathrm{a} \\
(61)\end{array}$ & $\begin{array}{c}1.03 \pm 0.14 \mathrm{c} \\
(-86)\end{array}$ & $\begin{array}{c}6.98 \pm 0.80 \mathrm{~b} \\
(-36.71)\end{array}$ & $\begin{array}{c}29.33 \pm 1.63 \mathrm{~d} \\
(-49)\end{array}$ & $\begin{array}{c}78 \pm 3.74 \mathrm{~b} \\
\quad(-9)\end{array}$ \\
\hline
\end{tabular}

Results are presented as mean \pm SEM. Means having different letters in the same column are significantly different $(\mathrm{P}<0.05)$ according to Duncan's Multiple Range Test. \% increase/decrease as compared with control $(0 \%)$ is presented in parenthesis, rounded off to nearest whole number

\section{Effect of Delonix regia aqueous leaf extract on early seedling growth of field bindweed and wheat}

Aqueous leaf extract of Delonix regia inhibited root and shoot length and seedling dry weight of field bindweed more than wheat (Table 2). Delonix regia aqueous leaf extract at $5 \%$ reduced root and shoot length of field bindweed by $42 \%$ and $45 \%$ 
respectively as compared with control, while root and shoot length of wheat was decreased by only $4 \%$ and $5 \%$ respectively as compared with control (Table 2). Similarly, seedling dry weight was decreased by $52 \%$ in field bindweed as compared with control, while it was statistically at par with control in wheat at 5\% leaf extract concentration (Table 2). Highest concentration (10\%) further decreased root and shoot length and seedling dry weight of field bindweed and wheat; however, it was less phytotoxic to wheat than field bindweed (Table 2).

Table 2. Effect of Delonix regia aqueous leaf extract on seedling growth of field bindweed and wheat

\begin{tabular}{c|c|c|c|c|c|c}
\hline \multirow{2}{*}{$\begin{array}{c}\text { Leaf extract } \\
\text { concentration } \\
(\%)\end{array}$} & \multicolumn{2}{|c|}{ Root length $(\mathbf{c m})$} & \multicolumn{2}{c|}{ Shoot length $(\mathbf{c m})$} & \multicolumn{2}{c}{ Seedling dry weight (mg) } \\
\cline { 2 - 7 } & $\begin{array}{c}\text { Field bind } \\
\text { weed }\end{array}$ & Wheat & $\begin{array}{c}\text { Field bind } \\
\text { weed }\end{array}$ & Wheat & $\begin{array}{c}\text { Field bind } \\
\text { weed }\end{array}$ & Wheat \\
\hline 0 & $5.08 \pm 0.00 \mathrm{a}$ & $5.02 \pm 0.02 \mathrm{a}$ & $5.93 \pm 0.43 \mathrm{a}$ & $5.3 \pm 0.07 \mathrm{~b}$ & $100 \pm 2.23 \mathrm{a}$ & $194 \pm 1.88 \mathrm{a}$ \\
2.5 & $5.04 \pm 0.05 \mathrm{a}$ & $4.92 \pm 0.04 \mathrm{a}$ & $5.80 \pm 0.04 \mathrm{~b}$ & $6 \pm 0.05 \mathrm{a}$ & $81.80 \pm 0.71 \mathrm{~b}$ & $191 \pm 3.73 \mathrm{a}$ \\
& $(-1)$ & $(-2)$ & $(-2)$ & $(14)$ & $(-18)$ & $(-2)$ \\
5.0 & $2.93 \pm 0.03 \mathrm{~b}$ & $4.80 \pm 0.04 \mathrm{c}$ & $3.29 \pm 0.05 \mathrm{c}$ & $5 \pm 0.07 \mathrm{~cd}$ & $48.20 \pm 1.65 \mathrm{c}$ & $192 \pm 2.56 \mathrm{a}$ \\
& $(-42)$ & $(-4)$ & $(-45)$ & $(-5)$ & $(-52)$ & $(-1)$ \\
7.5 & $2.20 \pm 0.00 \mathrm{c}$ & $3.83 \pm 0.03 \mathrm{~d}$ & $2.20 \pm 0.03 \mathrm{~d}$ & $5 \pm 0.03 \mathrm{c}$ & $44.60 \pm 1.96 \mathrm{c}$ & $160 \pm 4.09 \mathrm{~b}$ \\
& $(-57)$ & $(-24)$ & $(-63)$ & $(-4)$ & $(-55)$ & $(-17)$ \\
10 & $1.86 \pm 0.07 \mathrm{~d})$ & $3.09 \pm 0.03 \mathrm{e}$ & $2.22 \pm 0.02 \mathrm{~d}$ & $4.8 \pm 0.05 \mathrm{~d}$ & $33.60 \pm 1.57 \mathrm{~d}$ & $125 \pm 2.06 \mathrm{c}$ \\
& $(-63)$ & $(-38)$ & $(-63)$ & $(-8)$ & $(-66)$ & $(-36)$ \\
\hline
\end{tabular}

Results are presented as mean \pm SEM. Means having different letters in the same column are significantly different $(P<0.05)$ according to Duncan's Multiple Range Test. \% increase/decrease as compared with control $(0 \%)$ is presented in parenthesis, rounded off to nearest whole number

\section{Effect of Delonix regia aqueous leaf extract methanolic fractions on germination of field bindweed and wheat}

Ethyl acetate and lyophilized methanolic fractions of Delonix regia aqueous leaf extract significantly affected time to start germination, mean germination time and germination index in field bindweed, while these germination indices were not significantly affected in wheat (Table 3). At $1000 \mathrm{ppm}$ ethyl acetate fraction, time to start germination and mean germination time of filed bindweed was reduced by $30 \%$ and $13 \%$ respectively as compared with control, while there was no significant reduction in the two germination indices of wheat with application of the same treatment (Table 3). Likewise, germination index and final germination was inhibited by $64 \%$ and $48 \%$ respectively as compared with control in field bindweed at $1000 \mathrm{ppm}$ ethyl acetate fraction of Delonix regia, while germination index and final germination of wheat were not significantly affected at this concentration.

\section{Effect of Delonix regia aqueous leaf extract methanolic fractions on seedling growth of field bindweed and wheat}

All concentrations of ethyl acetate and lyophilized fractions of Delonix regia leaf extract significantly inhibited root and shoot length and seedling dry weight of field bindweed, while these parameters were only significantly reduced at higher concentrations of leaf extract in wheat (Table 4). At 1000 ppm ethyl acetate fraction, root length, shoot length and seedling dry weight of field bindweed was inhibited by 


$$
-516 \text { - }
$$

$59 \%, 46 \%$ and $46 \%$ respectively as compared with control. Root and shoot length and seedling dry weight was not significantly affected in wheat at $1000 \mathrm{ppm}$ ethyl acetate fraction (Table 4).

Table 3. Effect of Delonix regia leaf extract methanolic fractions on germination of field bindweed and wheat

\begin{tabular}{|c|c|c|c|c|c|c|c|c|c|}
\hline \multirow{2}{*}{$\begin{array}{l}\text { Name of } \\
\text { fraction }\end{array}$} & \multirow{2}{*}{$\begin{array}{c}\text { Concentration } \\
(\text { ppm })\end{array}$} & \multicolumn{2}{|c|}{$\begin{array}{c}\text { Time to start } \\
\text { germination (days) }\end{array}$} & \multicolumn{2}{|c|}{$\begin{array}{c}\text { Mean germination time } \\
\text { (days) }\end{array}$} & \multicolumn{2}{|c|}{ Germination index } & \multicolumn{2}{|c|}{ Final germination $(\%)$} \\
\hline & & $\begin{array}{c}\text { Field bind } \\
\text { weed }\end{array}$ & Wheat & $\begin{array}{c}\text { Field bind } \\
\text { weed }\end{array}$ & Wheat & $\begin{array}{c}\text { Field bind } \\
\text { weed }\end{array}$ & Wheat & $\begin{array}{c}\text { Field bind } \\
\text { weed }\end{array}$ & Wheat \\
\hline Control & 0 & $2.0 \pm 0.0 \mathrm{~cd}$ & $1 \pm 0.0^{\mathrm{NS}}$ & $2.97 \pm 0.20 \mathrm{~cd}$ & $1.43 \pm 0.11^{\mathrm{NS}}$ & $6.13 \pm 0.60 \mathrm{abc}$ & $12.5 \pm 0.92^{\mathrm{NS}}$ & $56.00 \pm 1.63 \mathrm{a}$ & $96 \pm 2.45 \mathrm{a}$ \\
\hline \multirow{5}{*}{ Ethyl acetate } & 50 & $\begin{array}{c}1.60 \pm 0.24 \mathrm{~d} \\
(-20)\end{array}$ & $\begin{array}{c}1 \pm 0.0 \\
(0)\end{array}$ & $\begin{array}{c}3.04 \pm 0.10 \mathrm{~cd} \\
\text { (2) }\end{array}$ & $\begin{array}{l}1.64 \pm 0.20 \\
(15)\end{array}$ & $\begin{array}{l}6.52 \pm 0.55 \mathrm{ab} \\
(6)\end{array}$ & $\begin{array}{l}12.97 \pm 1.13 \\
(4)\end{array}$ & $\begin{array}{c}54.66 \pm 2.49 \mathrm{a} \\
(-2)\end{array}$ & $\begin{array}{l}94 \pm 2.45 \mathrm{ab} \\
(-2)\end{array}$ \\
\hline & 500 & $\begin{array}{c}1.80 \pm 0.20 \mathrm{~d} \\
(-10)\end{array}$ & $\begin{array}{l}1 \pm 0.0 \\
(0)\end{array}$ & $\begin{array}{c}2.98 \pm 0.11 \mathrm{~cd} \\
(0)\end{array}$ & $\begin{array}{l}1.44 \pm 0.21 \\
\quad(1)\end{array}$ & $\begin{array}{c}5.70 \pm 0.12 \mathrm{abcd} \\
(-7)\end{array}$ & $\begin{array}{c}11.67 \pm 1.16 \\
(-7)\end{array}$ & $\begin{array}{c}44.00 \pm 1.63 \mathrm{c} \\
(-21)\end{array}$ & $\begin{array}{l}94 \pm 3.20 \mathrm{ab} \\
\quad(-2)\end{array}$ \\
\hline & 1000 & $\begin{array}{c}2.60 \pm 0.24 \mathrm{c} \\
(30)\end{array}$ & $\begin{array}{c}1 \pm 0.0 \\
(0)\end{array}$ & $\begin{array}{c}3.37 \pm 0.11 \mathrm{~cd} \\
(13)\end{array}$ & $\begin{array}{l}1.63 \pm 0.19 \\
(14)\end{array}$ & $\begin{array}{l}2.20 \pm 0.25 \mathrm{ef} \\
(-64)\end{array}$ & $\begin{array}{l}13.27 \pm 1.31 \\
(6)\end{array}$ & $\begin{array}{c}29.33 \pm 1.63 \mathrm{~d} \\
(-48)\end{array}$ & $\begin{array}{l}94 \pm 2.45 \mathrm{ab} \\
\quad(-2)\end{array}$ \\
\hline & 2000 & $\begin{array}{c}3.20 \pm 0.20 \mathrm{~b} \\
(60)\end{array}$ & $\begin{array}{c}1 \pm 0.0 \\
(0)\end{array}$ & $\begin{array}{l}4.01 \pm 0.21 b \\
\quad(35)\end{array}$ & $\begin{array}{l}1.43 \pm 0.20 \\
\quad(0)\end{array}$ & $\begin{array}{l}1.83 \pm 0.20 \mathrm{ef} \\
(-17)\end{array}$ & $\begin{array}{c}11.10 \pm 1.19 \\
(-11)\end{array}$ & $\begin{array}{c}25.33 \pm 1.33 \mathrm{de} \\
(-55)\end{array}$ & $\begin{array}{c}88 \pm 3.74 a b c \\
(-8)\end{array}$ \\
\hline & 4000 & $\begin{array}{c}4.20 \pm 0.20 \mathrm{a} \\
(110)\end{array}$ & $\begin{array}{c}1 \pm 0.0 \\
(0) \\
\end{array}$ & $\begin{array}{c}4.57 \pm 0.16 \mathrm{a} \\
(54) \\
\end{array}$ & $\begin{array}{c}1.67 \pm 0.10 \\
(17)\end{array}$ & $\begin{array}{c}1.06 \pm 0.17 \mathrm{f} \\
(-83)\end{array}$ & $\begin{array}{c}10.57 \pm 0.63 \\
(-15) \\
\end{array}$ & $\begin{array}{c}22.66 \pm 1.63 \mathrm{e} \\
(-60)\end{array}$ & $\begin{array}{c}80 \pm 4.46 \mathrm{c} \\
(-17) \\
\end{array}$ \\
\hline \multirow{5}{*}{ Lyophilized } & 50 & $\begin{array}{c}1.80 \pm 0.20 \mathrm{~d} \\
(-10)\end{array}$ & $\begin{array}{c}1 \pm 0.0 \\
(0)\end{array}$ & $\begin{array}{c}3.07 \pm 0.12 \mathrm{~cd} \\
\text { (3) }\end{array}$ & $\begin{array}{c}1.33 \pm 0.13 \\
(-7)\end{array}$ & $\begin{array}{c}6.11 \pm 0.66 \mathrm{abc} \\
(-0)\end{array}$ & $\begin{array}{c}11.50 \pm 0.89 \\
(-8)\end{array}$ & $\begin{array}{c}53.33 \pm 0.11 \mathrm{a} \\
(-5)\end{array}$ & $\begin{array}{l}96 \pm 2.45 \mathrm{a} \\
\quad(0)\end{array}$ \\
\hline & 500 & $\begin{array}{c}2.00 \pm 0.0 \mathrm{~cd} \\
(0)\end{array}$ & $\begin{array}{c}1 \pm 0.0 \\
(0)\end{array}$ & $\begin{array}{c}3.18 \pm 0.03 \mathrm{~cd} \\
(7)\end{array}$ & $\begin{array}{c}1.42 \pm 0.11 \\
(-1)\end{array}$ & $\begin{array}{c}5.62 \pm 0.18 \mathrm{abcd} \\
(-8)\end{array}$ & $\begin{array}{c}12.40 \pm 0.73 \\
(-0.8)\end{array}$ & $\begin{array}{c}46.66 \pm 0.10 \mathrm{bc} \\
(-17)\end{array}$ & $\begin{array}{l}96 \pm 2.45 \mathrm{a} \\
\quad(0)\end{array}$ \\
\hline & 1000 & $\begin{array}{c}2.20 \pm 0.20 \mathrm{~cd} \\
(10)\end{array}$ & $\begin{array}{l}1 \pm 0.0 \\
(0)\end{array}$ & $\begin{array}{c}3.23 \pm 0.20 \mathrm{~cd} \\
\text { (9) }\end{array}$ & $\begin{array}{c}1.64 \pm 0.10 \\
(15)\end{array}$ & $\begin{array}{c}4.83 \pm 0.51 \mathrm{~cd} \\
(-21)\end{array}$ & $\begin{array}{c}12.97 \pm 1.03 \\
\text { (4) }\end{array}$ & $\begin{array}{c}46.7 \pm 2.10 b c \\
(-17)\end{array}$ & $\begin{array}{c}92 \pm 3.74 \mathrm{ab} \\
(-4)\end{array}$ \\
\hline & 2000 & $\begin{array}{c}2.20 \pm 0.37 \mathrm{~cd} \\
(10)\end{array}$ & $\begin{array}{c}1 \pm 0.0 \\
(0)\end{array}$ & $\begin{array}{c}3.25 \pm 0.17 \mathrm{~cd} \\
(10)\end{array}$ & $\begin{array}{c}1.56 \pm 0.17 \\
\text { (9) }\end{array}$ & $\begin{array}{c}4.35 \pm 0.51 \mathrm{~d} \\
(-29)\end{array}$ & $\begin{array}{c}10.63 \pm 0.90 \\
(-15)\end{array}$ & $\begin{array}{c}46.66 \pm 2.10 b c \\
(-17)\end{array}$ & $\begin{array}{c}84 \pm 2.45 b c \\
(-13)\end{array}$ \\
\hline & 4000 & $\begin{array}{c}2.60 \pm 0.24 \mathrm{c} \\
(30)\end{array}$ & $\begin{array}{c}1 \pm 0.0 \\
(0) \\
\end{array}$ & $\begin{array}{c}3.43 \pm 0.12 \mathrm{c} \\
(14)\end{array}$ & $\begin{array}{c}1.87 \pm 0.22 \\
(31)\end{array}$ & $\begin{array}{c}2.87 \pm 0.72 \mathrm{e} \\
(-53)\end{array}$ & $\begin{array}{c}11.90 \pm 1.03 \\
(-5)\end{array}$ & $\begin{array}{c}29.33 \pm 3.39 d \\
(-48)\end{array}$ & $\begin{array}{c}84 \pm 2.45 \mathrm{dc} \\
(-13)\end{array}$ \\
\hline
\end{tabular}

Results are presented as mean \pm SEM. Means having different letters in the same column are significantly different $(P<0.05)$ according to Duncan's Multiple Range Test. \% increase/decrease as compared with control $(0 \%)$ is presented in parenthesis, rounded off to nearest whole number

Table 4. Effect of Delonix regia leaf extract methanolic fractions on seedling growth of field bindweed and wheat

\begin{tabular}{|c|c|c|c|c|c|c|c|}
\hline \multirow{2}{*}{$\begin{array}{l}\text { Name of } \\
\text { fraction }\end{array}$} & \multirow{2}{*}{\begin{tabular}{|c|}
$\begin{array}{c}\text { Concentration } \\
(\mathbf{p p m})\end{array}$ \\
\end{tabular}} & \multicolumn{2}{|c|}{ Root length (cm) } & \multicolumn{2}{|c|}{ Shoot length $(\mathrm{cm})$} & \multicolumn{2}{|c|}{ Seedling dry weight (mg) } \\
\hline & & Field bind weed & Wheat & Field bind weed & Wheat & Field bind weed & Wheat \\
\hline Control & 0 & $5.13 \pm 0.02 \mathrm{bcd}$ & $4.97 \pm 0.06 \mathrm{a}$ & $5.90 \pm 0.04 \mathrm{a}$ & $5.09 \pm 0.03 \mathrm{ab}$ & $105.2 \pm 3.24 \mathrm{a}$ & $194 \pm 3.29 \mathrm{bc}$ \\
\hline \multirow{5}{*}{ Ethyl acetate } & 50 & $\begin{array}{c}5.11 \pm 0.04 b c d \\
(-0)\end{array}$ & $\begin{array}{c}4.89 \pm 0.05 \mathrm{a} \\
(-2)\end{array}$ & $\begin{array}{c}5.85 \pm 0.05 \mathrm{a} \\
(-5)\end{array}$ & $\begin{array}{l}5.18 \pm 0.02 \mathrm{a} \\
(2)\end{array}$ & $\begin{array}{c}85.60 \pm 3.17 \mathrm{~cd} \\
(-19)\end{array}$ & $\begin{array}{c}201.4 \pm 2.89 \mathrm{~b} \\
\text { (4) }\end{array}$ \\
\hline & 500 & $\begin{array}{c}5.09 \pm 0.03 \mathrm{cde} \\
(-1)\end{array}$ & $\begin{array}{c}4.91 \pm 0.02 \mathrm{a} \\
(-1)\end{array}$ & $\begin{array}{c}5.79 \pm 0.06 \mathrm{a} \\
(-2)\end{array}$ & $\begin{array}{c}5.07 \pm 0.03 a b \\
(-0)\end{array}$ & $\begin{array}{c}60.0 \pm 1.97 \mathrm{~g} \\
(-43)\end{array}$ & $\begin{array}{c}200 \pm 2.37 \mathrm{~b} \\
\text { (3.) }\end{array}$ \\
\hline & 1000 & $\begin{array}{c}2.20 \pm 0.0 \mathrm{I} \\
(-59)\end{array}$ & $\begin{array}{c}4.89 \pm 0.04 a \\
(-2)\end{array}$ & $\begin{array}{c}3.17 \pm 0.03 \mathrm{~g} \\
(-46)\end{array}$ & $\begin{array}{c}5.02 \pm 0.04 \mathrm{abc} \\
(-1)\end{array}$ & $\begin{array}{c}46.20 \pm 2.13 \mathrm{~h} \\
(-56)\end{array}$ & $\begin{array}{c}201.4 \pm 2.83 \mathrm{~b} \\
\text { (4) }\end{array}$ \\
\hline & 2000 & $\begin{array}{l}1.85 \pm 0.04 \mathrm{j} \\
\quad(-64)\end{array}$ & $\begin{array}{c}3.31 \pm 0.02 b \\
(-33)\end{array}$ & $\begin{array}{l}2.29 \pm 0.02 \mathrm{~h} \\
(-61)\end{array}$ & $\begin{array}{l}3.74 \pm 0.06 \mathrm{f} \\
(-27)\end{array}$ & $\begin{array}{c}49.0 \pm 1.14 \mathrm{~h} \\
(-53)\end{array}$ & $\begin{array}{c}129 \pm 1.65 \mathrm{~g} \\
(-34)\end{array}$ \\
\hline & 4000 & $\begin{array}{c}1.19 \pm 0.02 \mathrm{k} \\
(-77)\end{array}$ & $\begin{array}{c}2.35 \pm 0.02 \mathrm{c} \\
(-53)\end{array}$ & $\begin{array}{l}2.23 \pm 0.01 \mathrm{~h} \\
(-62)\end{array}$ & $\begin{array}{c}3.17 \pm 0.03 g \\
(-38)\end{array}$ & $\begin{array}{c}32.20 \pm 1.07 \mathrm{i} \\
(-69)\end{array}$ & $\begin{array}{c}82.80 \pm 2.37 \mathrm{~h} \\
(-57)\end{array}$ \\
\hline \multirow{5}{*}{ Lyophilized } & 50 & $\begin{array}{l}5.33 \pm 0.07 a \\
\text { (4) }\end{array}$ & $\begin{array}{l}5.01 \pm 0.03 a \\
\text { (1) }\end{array}$ & $\begin{array}{c}5.87 \pm 0.02 \mathrm{a} \\
(-1)\end{array}$ & $\begin{array}{c}5.05 \pm 0.03 \mathrm{ab} \\
(-1)\end{array}$ & $\begin{array}{c}79.60 \pm 0.98 \mathrm{de} \\
(-24)\end{array}$ & $\begin{array}{c}140 \pm 1.39 f \\
(-28)\end{array}$ \\
\hline & 500 & $\begin{array}{c}5.28 \pm 0.09 \mathrm{ab} \\
\text { (3) }\end{array}$ & $\begin{array}{c}4.82 \pm 0.04 \mathrm{a} \\
(-3)\end{array}$ & $\begin{array}{l}5.94 \pm 0.05 \mathrm{a} \\
\text { (1) }\end{array}$ & $\begin{array}{c}4.96 \pm 0.03 \mathrm{bc} \\
(-3)\end{array}$ & $\begin{array}{c}71.60 \pm 0.20 \mathrm{f} \\
(-31)\end{array}$ & $\begin{array}{c}172 \pm 2.59 \mathrm{~d} \\
(-11)\end{array}$ \\
\hline & 1000 & $\begin{array}{c}4.29 \pm 0.02 \mathrm{~s} \\
(-16)\end{array}$ & $\begin{array}{c}4.90 \pm 0.02 \mathrm{a} \\
(-1)\end{array}$ & $\begin{array}{c}4.16 \pm 0.02 \mathrm{e} \\
(-29)\end{array}$ & $\begin{array}{c}4.99 \pm 0.06 \mathrm{bc} \\
(-2)\end{array}$ & $\begin{array}{c}71.40 \pm 2.06 \mathrm{ef} \\
(-32)\end{array}$ & $\begin{array}{c}153 \pm 2.80 \mathrm{e} \\
(-21)\end{array}$ \\
\hline & 2000 & $\begin{array}{c}3.18 \pm 0.02 \mathrm{~g} \\
(-38)\end{array}$ & $\begin{array}{c}4.73 \pm 0.02 \mathrm{a} \\
(-5)\end{array}$ & $\begin{array}{c}4.20 \pm 0.01 \mathrm{e} \\
(-29)\end{array}$ & $\begin{array}{c}4.89 \pm 0.03 c \\
(-4)\end{array}$ & $\begin{array}{c}69.60 \pm 0.40 \mathrm{f} \\
(-34)\end{array}$ & $\begin{array}{c}126.2 \pm 2.47 \mathrm{~g} \\
(-35)\end{array}$ \\
\hline & 4 & $\begin{array}{c}2.75 \pm 0.07 \mathrm{~h} \\
(-46) \\
\end{array}$ & $\begin{array}{c}3.30 \pm 0.02 b \\
(-33)\end{array}$ & $\begin{array}{c}3.52 \pm 0.19 \mathrm{f} \\
(-40)\end{array}$ & $\begin{array}{c}4.07 \pm 0.02 \mathrm{e} \\
(-20)\end{array}$ & $\begin{array}{c}50.20 \pm 1.46 \mathrm{~h} \\
(-52)\end{array}$ & $\begin{array}{c}152.2 \pm 4.06 \mathrm{e} \\
(-22)\end{array}$ \\
\hline
\end{tabular}

Results are presented as mean \pm SEM. Means having different letters in the same column are significantly different $(P<0.05)$ according to Duncan's Multiple Range Test. \% increase/decrease as compared with control $(0 \%)$ is presented in parenthesis, rounded off to nearest whole number 


$$
-517 \text { - }
$$

\section{Discussion}

Aqueous leaf extract and methanolic fractions (ethyl acetate and lyophilized) of Delonix regia had differential effect on germination and growth of field bindweed and wheat (Tables 1-4). Lower to medium concentrations of aqueous (2.5 and 5\%) and ethyl acetate and lyophilized methanolic fractions (50, 500 and $1000 \mathrm{ppm}$ ) of Delonix regia significantly inhibited germination and seedling growth of field bindweed, while these concentrations either did not significantly affect the germination and seedling growth of wheat or had very little inhibitory effect on it. Moreover, at lower extract concentrations there was a slight increase in germination and seedling growth of wheat. The slight increase in wheat growth might be due to plant growth promotive effect of allelochemicals on other plant species at lower concentrations (Akhtar et al., 2010; Jamil et al., 2009). Though higher Delonix regia leaf extract concentrations were phytotoxic for both field bindweed and wheat; nonetheless, phytotoxicity was very less on wheat as compared with field bindweed. The differential effect of allelopathic plant extracts on other plant species may be exploited to achieve selective phytotoxicity for weed management in field crops (Haq et al., 2010).

In the present study, the inhibitory effect of Delonix regia leaf extract may be attributed to variety of allelochemicals (chlorogenic acid, 4-hydroxybenzoic, 3,4dihydroxybenzoic, 3,5-dinitrobenzoic, L-azetidine-2-carboxylic 3,4dihydroxybenzaldehyde, 3,4-dihydroxycinnamic and gallic acid) reported in it (Chou and Leu, 1992). The inhibitory effect of allelochemicals in plant extracts have been well documented (Khaliq et al., 2012; Mushtaq et al., 2010, 2013). The phytotoxic effect of plant extracts is species-specific and concentration-dependent (Haq et al., 2010; Mushtaq et al., 2013). The presence of variety of allelochemicals in plant extract may act in synergistic way to inhibit growth of one plant species while their effect might be neutral or growth promotive on other plant species (Fujita and Kubo, 2003; Inderjit et al., 2002). Though aqueous extract as well as ethyl acetate and lyophilized methanolic fractions showed phytotoxicity in the bioassays; however, aqueous extract and ethyl fraction were more phytotoxic. Aqueous and ethyl acetate allelopathic plant extracts have been reported to have strong phytotoxic effects (Araniti et al., 2013; Mushtaq et al., 2010). Extraction of compounds by both aqueous and organic solvent fractions indicates that Delonix regia leaves contain diverse type of allelochemicals belonging to different chemical groups which enhance allelopathic potential of the plant.

\section{Conclusion}

Lower to moderate concentrations of Delonix regia leaf extract are phytotoxic against field bindweed, while safe on wheat crop. However, further studies may be carried out to evaluate the selective phytotoxic action of Delonix regia leaf extract against field bindweed and other weeds in various crops under field conditions. Delonix regia is a plant grown in many regions of the world. Its leaves are easily available and may be used to develop eco-friendly and selective bioherbicides in future especially for broad leaf weeds. 


$$
-518-
$$

\section{REFERENCES}

[1] Akhtar, H., Kausar, A., Akram, M., Cheema, Z. A., Ali, I., Mushtaq, M. N. (2010): Effects of Dalbergia sissoo Roxb. leaf extract on some associated crop species of agroforestry. - Allelopath. J. 25: 221-226.

[2] Aktar, M. W., Sengupta, D., Chowdhury, A. (2009): Impact of pesticides use in agriculture: their benefits and hazards. Interdiscip. - Toxicol. 2: 1-12.

[3] Albuquerque, M. B., dos Santos, R. C., Lima, L. M., Melo Filho, P. de A., Nogueira, R. J. M. C., da Câmara, C. A. G., de Rezende Ramos, A. (2011): Allelopathy, an alternative tool to improve cropping systems. A review. - Agron. Sustain. Dev. 31: 379-395.

[4] Araniti, F., Lupini, A., Sorgonà, A., Conforti, F., Marrelli, M., Statti, G. A., Menichini, F., Abenavoli, M. R. (2013): Allelopathic potential of Artemisia arborescens: Isolation, identification and quantification of phytotoxic compounds through fractionation-guided bioassays. - Nat. Prod. Res. 27: 880-887.

[5] Black, I. D., Matic, R., Dyson, C. B. (1994): Competitive effects of field bindweed (Convolvulus arvensis, L.) in wheat, barley and field peas. - Plant Prot. Q. 9: 12-14.

[6] Cheng, F., Cheng, Z. (2015): Research progress on the use of plant allelopathy in agriculture and the physiological and ecological mechanisms of allelopathy. - Front. Plant Sci. 6: 1020.

[7] Chou, C.-H., Leu, L.-L. (1992): Allelopathic substances and interactions of Delonix regia (Boj) Raf. - J. Chem. Ecol. 18: 2285-2303.

[8] De, A., Bose, R., Kumar, A., Mozumdar, S. (2014): Worldwide Pesticide Use. - In: De, A., Bose, R., Kumar, A., Mozumdar, S. (eds.) Targeted Delivery of Pesticides Using Biodegradable Polymeric Nanoparticles. Springer India, New Delhi.

[9] Fujita, K., Kubo, I. (2003): Synergism of polygodial and trans-cinnamic acid on inhibition of root elongation in lettuce seedling growth bioassays. - J. Chem. Ecol. 29: 2253-2262.

[10] Haq, R. A., Hussain, M., Cheema, Z. A., Mushtaq, M. N., Farooq, M. (2010): Mulberry leaf water extract inhibits bermudagrass and promotes wheat growth. - Weed Biol. Manag. 10: 234-240.

[11] Heap, I. (2018): The international survey of herbicide resistant weeds. http://www.weedscience.org (accessed 08.01.2018).

[12] Holm, L. G., Plucknett, D. L., Pancho, J. V, Herberger, J. P. (1977): The World's Worst Weeds: Distribution and Biology. - University Press of Hawaii., Hawaii.

[13] Inderjit, Streibig, J. C., Olofsdotter, M. (2002): Joint action of phenolic acid mixtures and its significance in allelopathy research. - Physiol. Plant. 114: 422-428.

[14] Iqbal, J., Cheema, Z. A., Mushtaq, M. N. (2009): Allelopathic crop water extracts reduce the herbicide dose for weed control in cotton (Gossypium Hirsutum). - Int. J. Agric. Biol. 11: 7 .

[15] Jamil, M., Cheema, Z. A., Mushtaq, M. N., Farooq, M., Cheema, M. A. (2009): Alternative control of wild oat and canary grass in wheat fields by allelopathic plant water extracts. - Agron. Sustain. Dev. 29: 475-482.

[16] Khaliq, A., Matloob, A., Aslam, F., Mushtaq, M. N., Khan, M. B. (2012): Toxic action of aqueous wheat straw extract on horse e purslane. - Planta Daninha 30: 269-278.

[17] Mushtaq, M., Cheema, Z., Khaliq, A. (2010): Effects of mixture of allelopathic plant aqueous extracts on Trianthema portulacastrum L. weed. - Allelopath. J. 25: 205-212.

[18] Mushtaq, M. N., Sunohara, Y., Matsumoto, H. (2013): L-DOPA inhibited the root growth of lettuce by inducing reactive oxygen species generation. - Weed Biol. Manag. 13: 129134.

[19] Orwa, C., Mutua, A., Kindt, R., Jamnadass, R., Anthony, S. (2009): Agroforestree database: a tree reference and selection guide version 4.0. http://www.worldagroforestry.org (accessed 08.05.2018). 


$$
-519-
$$

[20] Perveen, S., Yousaf, M., Zahoor, A. F., Rasool, N., Jabber, A. (2014): Extraction, isolation, and identification of various environment friendly components from cock's comb (Celosia argentea) leaves for allelopathic potential. - Toxicol. Environ. Chem. 96: 1523-1534.

[21] Polyrakis, I. T. (2009): Environmental Pollution from Pesticides. - In: Costa, R., Kristbergsson, K. (eds.) Predictive Modelling and Risk Assessment. Springer US, Boston, MA, pp. 201-224.

[22] Ranal, M. A., Santana, D. G. de (2006): How and why to measure the germination process? - Brazilian J. Bot. 29: 1-11.

[23] Sheng, Z., Li, J., Li, Y. (2012): Optimization of ultrasonic-assisted extraction of phillyrin from Forsythia suspensa using response surface methodology. - J. Med. Plants Res. 6: 1633-1644.

[24] Skinner, K., Smith, L., Rice, P. (2000): Using noxious weed lists to prioritize targets for developing weed management strategies. - Weed Sci. 48: 640-644. 